\title{
OBESITY EPIDEMIC IN SOUTH ASIA, TIME FOR CHANGE.......
}

\author{
M Weerakkody ${ }^{1}$, D Karuppiah ${ }^{2}$ \\ 1 Diabetes and Endocrine unit, Teaching Hospital Karapitiya, Galle. \\ ${ }^{2}$ Diabetes and Endocrine unit, Teaching Hospital Batticaloa.
}

Obesity has become one of the major public health concerns worldwide. A number of studies have reported that with each surge in weight, there is an increase in the risks for coronary heart disease, type 2 diabetes, cancers, hypertension, dyslipidaemia, stroke, sleep apnea, respiratory problems, osteoarthritis, and gynaecological problems. Although traditionally considered to be a problem associated with the developed world, with the recent economic, demographical, cultural and environmental changes, the developing world too is increasingly being burdened with the same issue. The South Asian region, with its number of diverse countries has not been spared of the problem, and has the additional issue of tackling with both under and over nutrition.

While low socioeconomic status has been associated with a higher prevalence of obesity and chronic diseases in developed countries, studies in developing nations have shown a positive socioeconomic status obesity relationship with increasing income level of such countries (1). Worldwide, the prevalence of obesity among women is also the highest in upper middle-income countries (1). Recent studies have demonstrated that the prevalence of obesity and the other related cardiovascular risk factors is high in the South Asian region, and is on the rise. Studies have revealed that the prevalence of obesity in India ranges between $20.8 \%$ to $50.1 \%$ in urban areas and is high as $32 \%$ in certain rural areas (2). In Sri Lanka, Katulanda et al have demonstrated that, according to the proposed World Health Organization cut-off body mass index values for Asians, the percentage of Sri Lankan adults in the overweight, obese and centrally obese categories were $25.2 \%, 9.2 \%$ and $26.2 \%$, respectively (3). Amongst children, again going by the global trends, there is a secular trend in the prevalence of obesity. Recent studies have quoted a prevalence of obesity as high as $29 \%$ amongst school children in certain Indian cities (2). However, the same region is also burdened by under nutrition, and it is estimated that more than half of the world's underweight children live in South Asia (4).

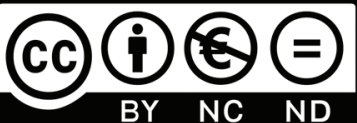

"Nutrition transition," termed as a combination of improved access to food and decreased physical activity level has been identified to be the prime risk factor for the increasing prevalence of overweight and chronic metabolic diseases in the developing countries, and is probably the cause of this problem in South Asia as well. Although initially such dietary changes and emergence of obesity was confined to higher socioeconomic strata of the populations among developing countries, more recent trends demonstrate a shift in the prevalence from the higher to the lower socioeconomic level (1). Nutritionists now believe that this nutrition transition is not merely due the change in the diet and physical activity, but is also the improvement in economic, social and environmental factors that is shaping the landscape of modern living as never before (4). In keeping with the global trends, South Asian nations too, have experienced a period of unprecedented economic growth, higher income level, provision of labor-saving technologies, and a significant reduction in the number of people living in extreme poverty. The global dietary transition, which is that of a shift from a diet with a higher proportion of carbohydrate based foods such as cereal grains (rice, wheat, maize), vegetables (leaves, roots, legumes) and low animal products (meat, egg, milk) to one which is lower in carbohydrate and higher in animal-based food with high sugar and caloric content and larger amount of processed food (sweets, soft drinks) has not spared the south Asian region as well. India, with around $35 \%$ of total population living on vegetarian diet has experienced a doubling in total poultry meat consumption since 2000, while in Pakistan's total meat consumption has increased by $130 \%$ during the same period (4). Increased import of food, advancement in local food technology and in food marketing and processing industry have greatly increased the availability of processed food products, even in the remote rural areas where majority of the South Asians live. Urbanization is thought to be one major cause for the change in these dietary habits. Nepal is leading the race of urbanization in South Asia with a rate of $4.9 \%$ while that for Sri Lanka is the lowest $(0.7 \%)$ (4). Furthermore, the number of people involved in 
service sector employment, as opposed to agriculture based jobs is also on the rise, and this is a major factor that leads people to move into urban areas. In Sri Lanka, service oriented employment rate has increased from $27.9 \%$ to $38.4 \%$ in the period from 1985 to 2005 whereas agriculture based employment has decreased from $49.3 \%$ to $30.7 \%$ (4). With increase in urbanization, there is more access to processed food, less time to prepare food at home and to engage in physical activity. With urbanization, there's more participation of women in the labour force, and it is likely that families may need to resort to consuming processed food rather than homemade traditional food, due to time constraints. Improvement in food technology, international trade and transport has led to the inflow of "international" food into countries, which are mostly processed food high in calories and saturated fat. This has changed the consumer market from one that consisted of mainly raw food and traditional food to one which mostly consists of processed ready-to-eat food. It is interesting to note India is the second largest (after Brazil) producer and largest consumer of sugar in the world (5).

Low physical activity which is another major contributor to obesity is also associated with low socio-economic status. Low physical activity may be due to less energy expenditure in farming and agricultural activities, increase in the number of occupations that require minimal amounts of energy expenditure and more time spent sedentarily during leisure time. Urbanization again, has created a number of office based jobs and reduced the amount of space that's available for physical activity such as safe walkways, bicycle paths, and playgrounds. Increase in time in front of the television has not only been associated with lack of physical activity, but also with increase in the consumption of unhealthy food in front of the television, which is also influenced by adverse nutrition messages in television commercials. Studies have constantly demonstrated that low socio-economic status is associated with low physical activity level. Studies reveal that physical activity is significantly lower in Asians when compared to their western counterparts. Data from the Health Survey from England found that south Asians in England were 60\% less likely to engage in physical activity than Caucasian living in the same region (6). A systematic review on physical inactivity amongst South Asians revealed that $18.5 \%-88.4 \%$ of Indians, $60.1 \%$ of Pakistanis and $11 \%-31.8 \%$ of Sri Lankans were physically inactive(6). However, contrary to results from other studies, which showed that lower economic status was associated with less amount of physical activity, this review revealed that skilled workers and professionals were more physically inactive and higher education was significantly associated with physical inactivity (6). Several studies have also revealed that physical inactivity is more in South Asia women than men, likely due to cultural restrictions in certain ethnic groups and the traditional role of women in household work and taking care of their families, which may leave them with very little time for recreational activities $(6,7$, 8). A recent study in Sri Lanka involving both rural and urban women in 20 to 45 year age group revealed that the prevalence of overweight, obesity and abdominal obesity was $38 \%, 34 \%$ and $45 \%$ respectively, which was higher than what was previously observed (9). In the same study, more than half of the women were categorized as being sedentary, and none of them were involved in any sport activities. Carbohydrates were the highest contributor to energy intake (70\%), and nearly $70 \%$ were consuming above the upper cut-off of recommendations for the amount of daily starch portions. There was a positive significant correlation between percentage of energy contributed from carbohydrate and waist circumference.

Over the years a number of interventions have been initiated to mitigate obesity, but with little success. The South Asian region, with its double burden of communicable and non-communicable diseases, need vigorous involvement of governments, nongovernmental organizations and media to combat this problem. Promotion of school based health and nutrition programmes to control childhood obesity is also an area that has been promoted by policymakers and researchers, which may be successful in the South Asian region, as South Asians have the highest number of children in the primary schools worldwide (1). Sri Lanka, in the recent past has initiated a number of such programmes, with the involvement of both the government and non-governmental organizations. Development of public outdoor areas such as parks and jogging tracts for recreational activities, promotion of exercise programmes within Government offices (a health promotion day per week), implementation of school canteen policies, promotion of school health clubs, introducing a coding policy to indicate the sugar level in sweetened beverages and the introduction of 
Healthy Lifestyle clinics through the public health system are some of them. However, what Sri Lanka lacks is a national policy and strict regulations on the import and sale of unhealthy food, a national policy on the amount of sugar and fat that can be incorporated into locally manufactured food and regulations on advertisement of unhealthy food via social media. Furthermore, although there is a positive trend in development of safe recreational areas for exercise, we have not still managed to make them accessible to all Sri Lankans, especially for those in semi-urban and rural areas of the country. Although there are a number of school health promotional activities that have been implemented, we still haven't addressed one of the key issues that affects school children of this country, that is inadequacy of time and resources to engage in creational activities, due to being overburdened by academic activities in today's competitive society.

\section{REFERENCES}

1. Bhurosyan T, Jeewan R. Overweight and Obesity Epidemic in Developing Countries: A Problem with Diet, Physical Activity, or Socioeconomic Status? Scientific World Journal Volume 2014, Article ID 964236.

2. Misra A, Shrivastava U. Obesity and Dyslipidaemia in South Asians. Nutrients. 2013 Jul; (7): 2708-2733.

3. Katulanda P, Jayawardena M A, Sheriff M H. Prevalence of overweight and obesity in Sri Lankan adults. Obes Rev 2010 Nov; 11(11):751-6.

4. Bhishwajit B. Nutrition transition in South Asia: the emergence of non-communicable chronic diseases. F1000Research 2015, 4:8. Last updated: 06 JAN 2016.

5. USDA (United States Department of Agriculture) Foreign Agricultural Service Report 2014. Available online: http://www.fas.usda.gov/regions/south-andcentral-asia/india.

6. Ranasinghe $\mathrm{C}$, Ranasinghe $\mathrm{P}$, Jayawardena $\mathrm{R}$ et al. Physical activity patterns among SouthAsian adults: A systematic review. International Journal of Behavioral Nutrition and Physical Activity 2013, 10:116.

7. Krishnan A, Shab B, Lal V et al. Prevalence of risk factors for non- communicable disease in rural area of Faridabad district of Haryana. Indian J of Public Health 2008, 52(3); 117-124.

8. Sugathan T N, Soman C R, Sankaranarayan K. Behavioural risk factors for noncommunicable diseases in Kerala India. Indian J Med R 2008, 127(6):555-563.
9. Rathnayake K, Roopasingham T, Dibley M. High carbohydrate diet and physical inactivity associated with central obesity among premenopausal housewives in Sri Lanka. BMC research notes $2014,7: 564$. 